\title{
Solitary wave solutions for high- order nonlinear Camassa-Holm equation
}

\author{
Chun-Huan Xiang ${ }^{1, a}$, Hong-Lei Wang ${ }^{2, b}$
}

${ }^{1}$ School of Public Health and Management, Chongqing Medical University, Chongqing, 400016, P.R. China

${ }^{2}$ College of medical informatics, Chongqing Medical University, Chongqing, 400016, P. R. China

aemail:w825900@163.com, bemail:w8259300@126.com

Keywords: nonlinear, Camassa-Holm equation, perturbation method, Solitary wave solutions Abstract: The solitary wave solutions to class of nonlinear fourth order variant of a generalized Camassa-Holm equation is investigated by employing the interesting perturbation method. The solitary wave solutions to Camassa-Holm equation is given in the form of Jacobi functions and expressed by the hyperbolic functions, the trigonometric functions with different modulus $k$. The numerical results for different types of solutions of the nonlinear Camassa-Holm equation are simply discussed.

\section{Introduction}

This perturbation method is more powerful to seek the exact solutions of the nonlinear partial differential equations in mathematical physics and investigation solutions for nonlinear equations is an important subject because they play important role in understanding the nonlinear problems. Recently, various powerful methods to deal with the evolution nonlinear equation are presented, such as Darboux transformation [1], the extended tanh-function method [2], the F-expansion method [3], homotopy analysis method [4], the Geometric Integrability method [5]. [6] presented numerical solutions of the time-dependent form and a discussion of the Camassa-Holm equation as a time-dependent Hamiltonian system. Cooper and Shepard [7] derived an approximate solitary wave solution to the Camassa-Holm equation using some variational functions. The high-order nonlinear Camassa-Holm equation[8-12] is investigated by employing the perturbation method in this paper.

\section{The nonlinear Camassa-Holm equation and perturbation method}

The Camassa-Holm equation was proposed by Camassa and Holm [13] as a model equation for unidirectional nonlinear dispersive waves in shallow water. The nonlinear Camassa-Holm equation can be rewritten as

$$
u_{t}-\mathrm{a} u_{x}+(b+1) u u_{x}-\alpha^{2}\left(u_{x x t}+u u_{x x x}+b u_{x} u_{x x}\right)_{x}+\gamma u_{x x x}=0
$$

where $\alpha, a, b, \gamma$ are known constants. Using the wave variable $\xi=x-c t$, where $c$ is wave speed, and proceeding as before we find

$$
-(c+a) u_{\xi}+(b+1) u u_{\xi}-\alpha^{2}\left(u u_{\xi \xi \xi}+b u_{\xi} u_{\xi \xi}\right)+\left(\alpha^{2} c+\gamma\right) u_{\xi \xi \xi}=0
$$

Integrating (2) with respect to $\xi$ and neglecting constants of integration we obtain

$$
-(c+a) u+\frac{1}{2}(b+1) u^{2}-\left(\alpha^{2} u-\alpha^{2} c-\gamma\right) u_{\xi \xi}-\frac{1}{2}(b-1) \alpha^{2} u_{\xi}^{2}=0
$$

The Eq.(3) will be dealed with the perturbation method to obtain the solitary wave solutions.

For a given nonlinear equation as the follow

$$
G\left(u, u_{x}, u_{t}, u_{x x}, u_{t t}, u_{x t}, \ldots\right)=0
$$

where $u_{x}=\partial u / \partial x, u_{x x}=\partial^{2} u / \partial x^{2}, u_{x t}=\partial^{2} u / \partial x \partial t, \ldots$ with the expression as $\xi=x-c t$, where $c$ denotes the wave speed, then $G$ leads to an ordinary differential equation.

$$
G\left(u, u_{\xi}, u_{\xi \xi}, u_{\xi \xi \xi}, \ldots\right)=0
$$


The perturbation expand is employed to obtain the approximation evolution solution for nonlinear equation.

$$
u(\xi)=a_{0}+a_{1} y+a_{2} y^{2}+\ldots
$$

where $a$ is small parameter, $y$ is the solitary functions, with the following the relations:

$$
\begin{aligned}
& \left(y_{x}\right)^{2}=m_{1}+m_{2} y^{2}+m_{3} y^{4} \\
& y_{x x}=m_{2} y+2 m_{3} y^{3}
\end{aligned}
$$

Here, $u(\xi)=a_{0}+a_{1} y$ is used in the Eq.(5) in this work. Substituting Eq. (5)-(7) into Eq.(3) and equating to zero the coefficients of all powers of $y$ yields a set of equations, the parameters are obtained .

\section{Numerical example}

We employ the perturbation method for the nonlinear equations (3), the useful expression (6) (7) is used. Substituting Eq. (5)-(7) into Eq. (3), and equating the coefficients of all powers of $y$ to zero, the first equations as follows:

$$
\begin{gathered}
-2 a a_{0}-2 c a_{0}+a_{0}{ }^{2}+b a_{0}{ }^{2}+m_{1} \alpha^{2} a_{1}^{2}-b m_{1} \alpha^{2} a_{1}^{2}=0 \\
-a a_{1}+m_{2} a_{1}-c a_{1}+m_{2} \alpha^{2} c a_{1}+a_{1} a_{0}+b a_{1} a_{0}-a_{0} m_{2} \alpha^{2} a_{1}=0 \\
2 m_{3} a_{1}+2 m_{3} \alpha^{2} c a_{1}-2 m_{3} \alpha^{2} a_{1}^{2}=0 \\
a_{1}^{2}+b a_{1}^{2}-m_{2} \alpha^{2} a_{1}^{2}-b m_{2} \alpha^{2} a_{1}^{2}=0 \\
3 m_{3} \alpha^{2} a_{1}^{2}+b m_{3} \alpha^{2} a_{1}^{2}=0
\end{gathered}
$$

Eq.(8)-(12) can be solved and obtain the relation between the parameters.

$$
\begin{aligned}
& m_{2} \alpha^{2}=1 \quad b=-3 \quad a_{0}=\left(c-a \alpha^{2}\right) / 3 \alpha^{2} \\
& (3 c-a) \alpha^{2}=-\gamma \\
a_{1}= & \sqrt{\left(c^{2}+a c \alpha^{2}-2 a^{2} \alpha^{4}+3 c \gamma \alpha^{2}-3 c a \alpha^{4}\right) / 18 m_{1} \alpha^{6}}
\end{aligned}
$$

The Solitary wave solutions for Eq.(1) is given in the Jacobi functions forms

$$
u=\frac{\left(c-a \alpha^{2}\right)}{3 \alpha^{2}}+\frac{\sqrt{\left(c^{2}+a c \alpha^{2}-2 a^{2} \alpha^{4}+3 c \gamma \alpha^{2}-3 c a \alpha^{4}\right)}}{18 m_{1} \alpha^{6}} \operatorname{sn}((x-c t), k)
$$

$$
\begin{aligned}
& u=\frac{\left(c-a \alpha^{2}\right)}{3 \alpha^{2}}+\frac{\sqrt{\left(c^{2}+a c \alpha^{2}-2 a^{2} \alpha^{4}+3 c \gamma \alpha^{2}-3 c a \alpha^{4}\right)}}{18 m_{1} \alpha^{6}} c n((x-c t), k), \\
& u=\frac{\left(c-a \alpha^{2}\right)}{3 \alpha^{2}}+\frac{\sqrt{\left(c^{2}+a c \alpha^{2}-2 a^{2} \alpha^{4}+3 c \gamma \alpha^{2}-3 c a \alpha^{4}\right)}}{18 m_{1} \alpha^{6}} d n((x-c t), k) \\
& u=\frac{\left(c-a \alpha^{2}\right)}{3 \alpha^{2}}+\frac{\sqrt{\left(c^{2}+a c \alpha^{2}-2 a^{2} \alpha^{4}+3 c \gamma \alpha^{2}-3 c a \alpha^{4}\right)}}{18 m_{1} \alpha^{6}} n d((x-c t), k) \\
& u=\frac{\left(c-a \alpha^{2}\right)}{3 \alpha^{2}}+\frac{\sqrt{\left(c^{2}+a c \alpha^{2}-2 a^{2} \alpha^{4}+3 c \gamma \alpha^{2}-3 c a \alpha^{4}\right)}}{18 m_{1} \alpha^{6}} s d((x-c t), k) \\
& u=\frac{\left(c-a \alpha^{2}\right)}{3 \alpha^{2}}+\frac{\sqrt{\left(c^{2}+a c \alpha^{2}-2 a^{2} \alpha^{4}+3 c \gamma \alpha^{2}-3 c a \alpha^{4}\right)}}{18 m_{1} \alpha^{6}} c d((x-c t), k)
\end{aligned}
$$

From the above equation (13)-(15), when $k \rightarrow 0$ or $k \rightarrow 1$, the Eq. (13)-(15) are reduced into trigonometric or hyperbolic solutions for Eq. (1)

$k \rightarrow 0$ 


$$
\begin{aligned}
& u=\frac{\left(c-a \alpha^{2}\right)}{3 \alpha^{2}}+\frac{\sqrt{\left(c^{2}+a c \alpha^{2}-2 a^{2} \alpha^{4}+3 c \gamma \alpha^{2}-3 c a \alpha^{4}\right)}}{18 m_{1} \alpha^{6}} \sin (x-c t) \\
& u=\frac{\left(c-a \alpha^{2}\right)}{3 \alpha^{2}}+\frac{\sqrt{\left(c^{2}+a c \alpha^{2}-2 a^{2} \alpha^{4}+3 c \gamma \alpha^{2}-3 c a \alpha^{4}\right)}}{18 m_{1} \alpha^{6}} \cos (x-c t), \\
& u=\frac{\left(c-a \alpha^{2}\right)}{3 \alpha^{2}}+\frac{\sqrt{\left(c^{2}+a c \alpha^{2}-2 a^{2} \alpha^{4}+3 c \gamma \alpha^{2}-3 c a \alpha^{4}\right)}}{18 m_{1} \alpha^{6}}
\end{aligned}
$$

$k \rightarrow 1$

$$
\begin{aligned}
& u=\frac{\left(c-a \alpha^{2}\right)}{3 \alpha^{2}}+\frac{\sqrt{\left(c^{2}+a c \alpha^{2}-2 a^{2} \alpha^{4}+3 c \gamma \alpha^{2}-3 c a \alpha^{4}\right)}}{18 m_{1} \alpha^{6}} \tanh (x-c t) \\
& u=\frac{\left(c-a \alpha^{2}\right)}{3 \alpha^{2}}+\frac{\sqrt{\left(c^{2}+a c \alpha^{2}-2 a^{2} \alpha^{4}+3 c \gamma \alpha^{2}-3 c a \alpha^{4}\right)}}{18 m_{1} \alpha^{6}} \sec h(x-c t), \\
& u=\frac{\left(c-a \alpha^{2}\right)}{3 \alpha^{2}}+\frac{\sqrt{\left(c^{2}+a c \alpha^{2}-2 a^{2} \alpha^{4}+3 c \gamma \alpha^{2}-3 c a \alpha^{4}\right)}}{18 m_{1} \alpha^{6}} \operatorname{sech}(x-c t)
\end{aligned}
$$

The simulation figure for Eq.(13)-(15) is shown in Fig. 1, the parameters are $k=0.7 ; a=0.2 ; \mathrm{c}=-0.3$; $\mathrm{m}_{1}=1, \mathrm{~m}_{2}=-\left(1+k^{2}\right), x \in(-20,20), \quad t \in(-20,20)$, respectively.
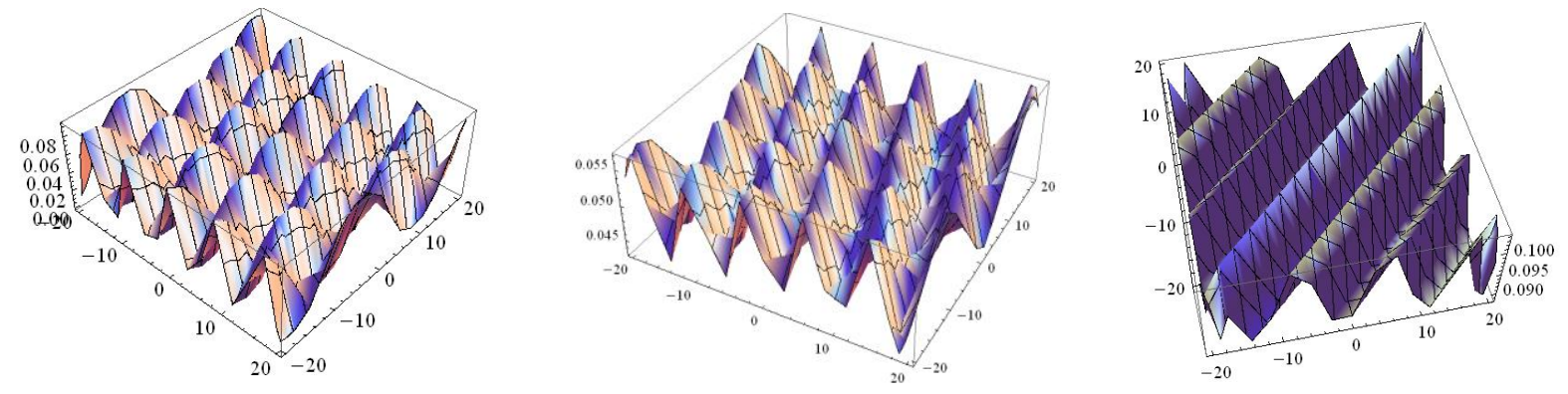

Fig.1 The simulation result is shown for Eq. (13)-(15)

The simulation figure for Eq.(16)-(18) is shown in Fig. 2, the parameters are $k=0.9 \backslash 0.1 \backslash 0.8 ; a=$ $0.2 \backslash 1 \backslash-1 ; \mathrm{c}=-0.3 ; \mathrm{m}_{1}=1, \mathrm{~m}_{2}=-\left(1+k^{2}\right), x \in(-35,35), \quad t \in(-35,35), x \in(-5,5), \quad t \in(-5,5)$,

$x \in(-35,35), \quad t \in(-35,35)$, respectively.
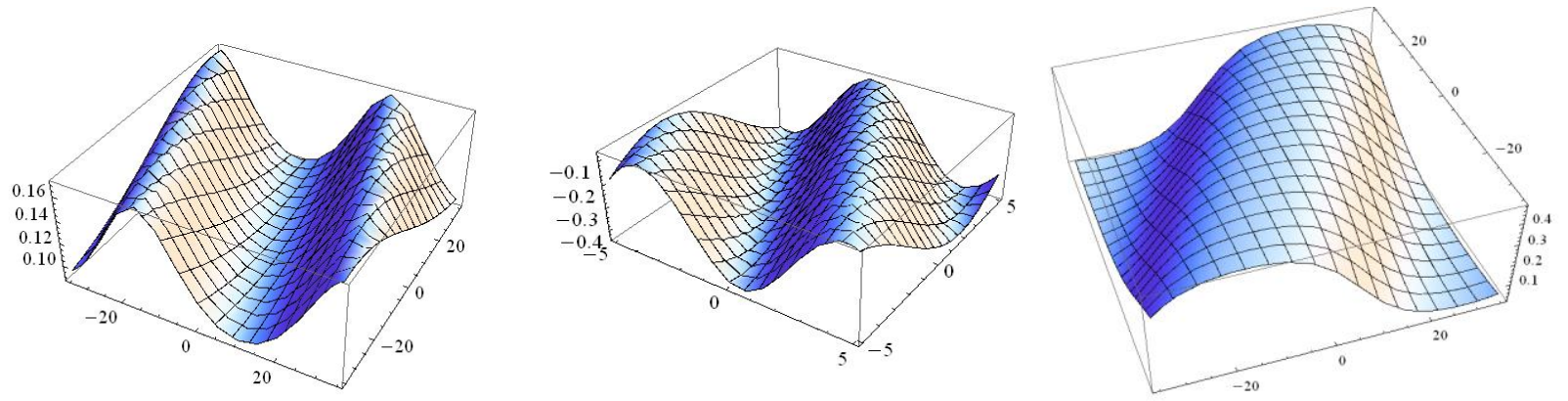

Fig.2 The simulation result is shown for Eq. (16)-(18) 


\section{Conclusions}

The nonlinear Camassa-Holm equation is investigated in this manuscript and obtained an excellent analytic approximation to the family of solitary solutions by employing the perturbation method, which performance provides us with a convenient way to control the convergence of approximation series. The simulation figures for the evolution solutions with some parameters are shown. We demonstrated the accuracy and efficiency of this method by solving for the considered nonlinear differential equation system.

\section{References}

[1] V.A. Matveev, M.A. Salle, Darboux transformations and solitons. Berlin, Heidelberg: Springer-Verlag. (1991).

[2] E. G. Fan, Extended tanh-function method and its applications to nonlinear equations. Phys. Lett. A .277:212-220 (2000).

[3] Y. B. Zhou, M. L. Wang, Y. M. Wang, Periodic wave solutions to a coupled KdV equations with variable coefficients. Phys. Lett. A. 308:31-37 (2003).

[4] S. Abbasbandy, E.J. Parkes, Solitary smooth hump solutions of the Camassa-Holm equation by means of the homotopy analysis method. Chaos Solitons \& Fractals · May 2008.

[5] R. H. Heredero and E. G. Reyes, Geometric Integrability of the Camassa-Holm Equation. International Mathematics Research Notices · January 2012.

[6] R. Camassa, D.D. Holm, J.M. Hyman, A new integrable shallow water equation, Adv. Appl. Mech. 31, 1-33, (1994).

[7] F. Cooper, H. Shepard, Solitons in the Camassa-Holm shallow water equation, Phys. Lett. A, 194 (4), 246-250, (1994).

[8] J. Lenells, Traveling wave solutions of the Camassa-Holm equation, J. Differential Equations 217 393-430, (2005)

[9] X. Fan and S. Li, Bifurcation on Smooth and Non-smooth Traveling Wave Solutions for a Generalized Two-component Camassa-Holm Equation, Journal of Scientific Research \& Reports 3(13): 1815-1827, (2014)

[10] M. Wei, Y. Gan, S. Tang, Bifurcations of Travelling Wave Solutions for the B(m,n) Equation. American Journal of Computational Mathematics, 4, 104-118, (2014)

[11] J. Rong, S. Tang, W. Huang, Bifurcations of travelling wave solutions for a class of nonlinear fourth order variant of a generalized Camassa-Holm equation. Commun. Nonlinear Sci. Numer. Simulat. 15, 3402-3417, (2010)

[12] J. Li, Y. Exact loop solutions, cusp solutions, solitary wave solutions and periodic wave solutions for the special CH-DP equation. Nonlinear Analysis: Real World Applications 10 , 2502-2507, (2009).

[13] R. Camassa, D.D. Holm, An integrable shallow water equation with peaked solitons, Phys. Rev. Lett. 71, 1661-1664, (1993). 